\title{
Ségocier la dépendance?
}

\author{
Alan Ouakrat \\ Maître de conférences \\ Université Sorbonne Nouvelle \\ IRMECCEN \\ France \\ alan.ouakrat@sorbonne-nouvelle.fr
}

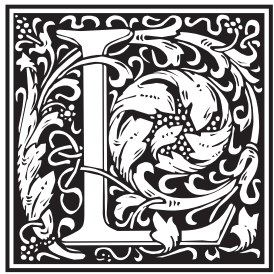

es plateformes participent à re-centraliser l'accès aux contenus pour les publics. En organisant l'accès à l'actualité, elles exercent un rôle méta-éditorial. Elles définissent une norme technique de sélection et de mise en visibilité des contenus à travers leurs algorithmes. Pour favoriser l'indexation de leurs contenus, les éditeurs sont fortement incités à respecter les critères considérés comme pertinents par la plateforme, sans y être formellement contraints (Badouard et al., 2016). La " menace de l'invisibilité " (Bucher, 2012) pourrait pénaliser ceux qui ne se conforment pas à la règle technique (Lessig, 1999). Or, la concurrence pour la visibilité est très forte sur internet. Elle détermine en grande partie l'accès aux revenus, ce qui engendre un rapport de dépendance réciproque mais asymétrique entre la presse et les plateformes. Devenues des infrastructures essentielles de la vie numérique en matière d'information et de communication (Plantin et al., 2016 ; Nechushtai, 2017), les plateformes sont des points de passage incontournables pour les éditeurs.

Notre étude analyse un moment particulier de l'affaire du droit voisin pour les éditeurs de presse, le contournement de la loi par Google. Par cet

Pour citer cet article, to quote this article, para citar este artigo :

Alan Ouakrat, « Négocier la dépendance? Google, la presse et le droit voisin. », Sur le journalisme, About journalism, Sobre jornalismo [En ligne, online], Vol 9, n 1 - 2020, 15 juin June 15 - 15 de juno.

URL : http://www.surlejournalisme.com/rev 
article, nous souhaitons éclairer les enjeux de la relation entre Google et les éditeurs de presse, tout en prenant en compte comment celle-ci est configurée par l'action publique. À cette fin, nous mobilisons des études qui ont principalement trait à trois domaines : les sciences de l'information et de la communication pour comprendre les enjeux spécifiques aux relations entre les plateformes et la presse ; les études en sciences et techniques (STS) pour saisir comment opèrent les plateformes et le code informatique pour modeler l'environnement et les possibilités d'action de leurs utilisateurs ; et enfin, des recherches au croisement du droit et de l'économie qui envisagent comment le pouvoir des plateformes peut être encadré par les États. Un certain nombre de travaux se sont penchés sur les relations entre les plateformes et les éditeurs de presse (Sire, 2013 ; Nielsen \& Ganter, 2017 ; Nechushtai, 2017 ; Bell \& Owen, 2017 ; Rashidian et al., 2018 ; Rashidian et al., 2019 ; Smyrnaios \& Rebillard, 2019). D’autres recherches ont exploré la rencontre du droit et de la technique dans la régulation de l'internet (Lessig, 1999 ; Goldsmith \& Wu, 2006 ; Dulong De Rosnay, 2016 ; Tréguer, 2019 ; Suzor, 2019). Des travaux se sont intéressés à la gouvernance par les plateformes, en cherchant à comprendre comment la règle technique opère à travers le code, les infrastructures et les algorithmes (Brown \& Marsden, 2013 ; Bucher, 2012 ; Suzor, 2019 ; Reidenberg, 1998 ; Grimmelman, 2005 ; Musiani, 2013 ; Helmond, 2015 ; Ziewitz, 2016). Dans cet article, nous souhaitons montrer comment les plateformes gouvernent le marché et les interactions avec leurs utilisateurs (Rieder \& Sire, 2014 ; Badouard et al., 2016), en l'occurrence les éditeurs de presse, et comment elles ont à négocier un compromis avec les États (Gorwa, 2019). Nous explorons ainsi une tension entre la régulation par les plateformes et la régulation des plateformes, qui nous semble particulièrement observable depuis l'affaire du droit voisin des éditeurs de presse. Celle-ci paraît emblématique d'un moment d'ajustement des rapports de pouvoir entre les États, les éditeurs et les plateformes. Par conséquent, nous interrogeons dans quelle mesure et à quelles conditions s'opère une négociation des rapports de dépendance entre les éditeurs et Google, médiée par la loi sur le droit voisin.

Pour répondre à notre problématique, nous revenons sur la relation qui unit Google aux éditeurs de presse. La dépendance aux plateformes des éditeurs de presse est duale, aussi bien économique qu'éditoriale. Ces derniers ont identifié les limites du marché publicitaire numérique pour le développement de leurs revenus, raison pour laquelle ils ont opéré un tournant stratégique vers l'abonnement payant. Google propose un accompagnement dans l'innovation éditoriale et commerciale dédié au journalisme numérique, auquel il consacre une initiative mondiale (Google News Initiative). Face à la captation de l'essentiel des revenus publicitaires par les plateformes, le nouveau droit voisin des éditeurs de presse propose de redistribuer la valeur économique en faveur des éditeurs ${ }^{1}$. Cette réponse juridique liée à la propriété intellectuelle et inscrite dans le cadre d'une directive européenne s'est heurtée au contournement de la règle de droit par Google. L'action contentieuse mené par les éditeurs de presse auprès de l'Autorité de la concurrence (Adlc) a conduit à rappeler à Google, dans une décision provisoire, la nécessité d'une négociation collective et transparente, devant conduire à la rémunération prévue par la loi. La première partie de cet article s'intéresse à la relation entre Google et les éditeurs de presse telle qu'elle est régulée par la plateforme. La seconde partie montre à travers l'affaire du droit voisin comment l'État régule la plateforme. Avec le nouveau droit voisin, il s'agit de passer d'une exploitation de fait à une exploitation de droit des contenus d'actualité.

\section{Méthodologie}

Les matériaux empiriques mobilisés sont les suivants :

- Une table-ronde dans le cadre du colloque " Marques, publicité et création audiovisuelle » que nous avons organisé à la Sorbonne Nouvelle en février 2018, réunissant des professionnels occupant des postes à responsabilité dans le secteur de la presse autour de la question «Que changent les alliances des éditeurs dans la data face aux GAFAM ?»;

- Deux entretiens menés en janvier et en mars 2020 avec des éditeurs de presse numérique dans le cadre du programme ANR Pluralisme de l'Information en Ligne (PIL) (ANR-17-CE27-0010) (20182021), conduits avec Lorreine Petters ;

- Une interview vidéo publique de Ludovic Blecher (head of innovation pour Google News Initiative) par Philippe Couve (Samsa.fr), 24 juillet 2019. "Google appuie les innovations médias en Afrique et au Moyen-Orient », 8mn29s.

https://www.youtube.com/watch?time_continue $=1$ $\& v=0 m W 4 Q 0 Z X m p 0 \&$ feature $=$ emb_title

- Une interview vidéo publique de David Dieudonné, directeur de Google News Lab France, en plateau avec l'EPJT aux assises du journalisme, 14 mars 2018, 4mn24s.

https://www.youtube.com/watch?v=ANHYNWulGQ8 
Une décision de l'Autorité de la Concurrence. Décision n ${ }^{\circ}$ 20-MC-01, 9 avril 2020, 72p.

https://www.autoritedelaconcurrence.fr/sites/default/files/integral_texts/2020-04/20mc01.pdf

\section{Une dépendance mutuelle mais asymétrique}

Google apporte un trafic considérable aux éditeurs que ceux-ci tentent ensuite de monétiser soit par de la publicité soit par le paiement direct des publics, notamment avec l'abonnement ${ }^{2}$. Dans cette partie, nous proposons d'analyser les avantages apportés par Google aux annonceurs par rapport à ceux offerts par les éditeurs de presse. Ces derniers envisagent désormais un tournant vers l'abonnement payant comme stratégie principale de développement de leurs revenus numériques. Pour Google, l'avenir du journalisme est indissociablement lié au sien, raison par laquelle la plateforme justifie son soutien à l'innovation éditoriale et commerciale dans le secteur de la presse.

\section{De la quête de monétisation publicitaire au tournant vers le payant}

Le succès publicitaire de la plateforme Google repose sur l'association étroite entre une stratégie de collecte des données des consommateurs et l'offre de solutions technologiques aux annonceurs et aux éditeurs. La clé de sa réussite réside dans le volume de données collectées par la quantité des publics rassemblés, la granularité de ces données et la fréquence de leur actualisation. Une valeur singulière est accordée aux services de Google par les annonceurs en raison de la combinaison de ces données avec des solutions publicitaires permettant de les exploiter. Si l'offre se révèle pertinente, c'est en raison de la capacité de Google à « activer » ces données, un avantage majeur par rapport aux éditeurs de presse.

«Il faut que [1]es gens reviennent sur mon site pour que je puisse les toucher. On oublie un élément qui est absolument essentiel, c'est l'activation ensuite de cette donnée. Il faut que la personne revienne sur mon site tous les jours, plusieurs fois par jour parce que j'ai beau l'avoir flagué comme un intentionniste automobile $^{3}$, [s'il] ne revient pas le lendemain et que c'est le lendemain que Mercedes veut faire sa campagne et ben on a raté. [...] Il y a deux choses dans la data dans le programmatique, c'est d'être en capacité de mettre un profil derrière une personne mais après c'est de retrouver cette personne sur nos sites. " (table-ronde, directrice data, Les Échos-Le Parisien, 08/02/18)
D’après Google, les éditeurs peuvent monétiser auprès des annonceurs l'attention des publics qu'il amène sur leurs espaces. Or, cela n'a rien d'évident tant Google participe dans le même temps à redéfinir le niveau d'attente et d'exigence des annonceurs dans la publicité numérique par ses technologies.

"Ils savent très bien qu'on ne peut pas se passer d'eux, il y a un rapport de forces. En plus, Google, ils sont présents sur la totalité du marché et ils contribuent drastiquement par ailleurs à faire baisser le prix de la monétisation de la publicité avec le programmatique [...] Ils tapent sur l'épaule et disent "On va vous aider, vous ne savez pas faire. On va vous apprendre à pêcher grâce à nos outils et à nos formations. On va former vos journalistes. Grâce à tout ça, on va vous apprendre à faire du business " (Directeur du pôle News Le Figaro et président du Geste, 23/01/20)

Pour les éditeurs, il s'agit de métiers, de compétences et d'un savoir-faire technologique à acquérir. Les recrutements favorisent le développement de ces compétences mais les éditeurs s'appuient souvent sur les outils de la plateforme pour la collecte, le traitement ou l'exploitation des données liées aux publics ou à la gestion des campagnes publicitaires.

"Il a fallu faire des choix technologiques, embaucher des profils qui sont des gens qu'on n'avait pas dans nos maisons pour pouvoir gérer ces technos, pour pouvoir travailler avec ET combattre les GAFA. Ce sont vraiment nos frenemies... Le Monde, en l'occurrence, on a tout le stack ${ }^{4}$ Google, l'ad-server pour servir nos publicités, un branchement avec leur ad-exchange ${ }^{5}$... Pour autant, nous n'avons pas pris leur data, c'est-à-dire que l'on fait des choses et pas d'autres. On utilise Google pour être notre fournisseur de technos, on pourrait l'utiliser plus, avoir plus de technos, avoir la totalité de la chaîne, ce que l'on n'a pas fait [...] C'est tout ce jeu de trouver comment on travaille avec eux. Il a fallu qu'on ait des nouveaux profils chez nous. Manager une relation avec Google, ca ne pouvait pas se faire avec les gens qui étaient initialement dans nos équipes. " (table-ronde, présidente MPublicité-Groupe Le Monde, 08/02/18)

Google et Facebook proposent des solutions d'achat d'espaces publicitaires intégrées. Une illustration en est le lancement de la Google Marke- 
ting Platform en 2018 qui centralise l'ensemble des outils publicitaires de Google (Allary et Ballusseau, 2018 ; Guignard, 2019). Il s'agit d'une solution agréable à utiliser pour les annonceurs, appuyée par le savoir-faire de Google en matière d'expérience utilisateur. De plus, ces services publicitaires répondent à une logique de guichet unique permettant de réduire les coûts de transaction pour les annonceurs. Par conséquent, les demandes et le niveau d'exigence de ces derniers ont été modelés par leur pratique des outils de Google. Cela les a conduits à inciter les éditeurs à collaborer avec la plateforme et à utiliser ses outils publicitaires pour faciliter le suivi des actions sur les consommateurs.

Les éditeurs ont d'abord tenté d'opposer leurs savoir-faire spécifiques en matière de publicité, notamment la connaissance des besoins des annonceurs et les relations commerciales de long terme entretenues avec les grandes marques. Toutefois, ils ont été débordés par le caractère transnational de l'offre publicitaire des géants de l'internet et l'harmonisation technique des normes de référence du commerce de publicité impulsée par Google. En imposant ses standards à l'activité publicitaire numérique, la plateforme a dépossédé les éditeurs de la maîtrise de leur relation commerciale avec les marques - bien qu'ils aient tenté de lancer des innovations liées à la mutualisation de leurs offres à travers des stratégies d'alliances (telles que Gravity ou Skyline) ou encore la défense d'un label éthique de publicité responsable (Digital Ad Trust). Le succès modeste de ces initiatives peut être imputé à leur périmètre comparativement limité (souvent national voire européen) et à leur caractère tardif, alors que les normes du marché s'étaient déjà déplacées. Elles sont apparues comme en décalage avec les possibilités offertes aux annonceurs par les plateformes. Par ailleurs, la relation entre Google et les éditeurs de presse concerne aussi la mise en visibilité éditoriale des contenus sur les services de la plateforme, qui passe notamment par l'optimisation du référencement (SEO) et, éventuellement, l'achat de liens sponsorisés pour accroître la visibilité de leurs contenus dans une stratégie de promotion de la marque éditoriale.

Le tournant vers l'abonnement payant numérique est une stratégie mise en œuvre par les éditeurs de presse face à des revenus publicitaires insuffisants pour financer l'activité de production éditoriale. Et ce, malgré les nombreux développements que les éditeurs ont réalisés dans le programmatique. Le paiement direct par les publics a toujours existé. Il est une source historique de revenus pour la presse imprimée, ainsi que le moyen le plus sûr et le plus stable de rémunération (Arrese, 2015). Pourtant, en France, c'est essentiellement à partir de 2016 que des investissements stratégiques significatifs sont réalisés en faveur de l'abonnement payant.

«Une partie du combat pour nous c'est de produire de plus en plus de contenus exclusifs de très très grande qualité [et de] savoir en monétiser une partie. Une partie de la solution c'est les abonnements payants, c'est-à-dire que l'on pense que le modèle ne sera pas que publicitaire. " (MPublicité, 08/02/18)

«Sous la pression aussi de cette arrivée du programmatique, il y a eu effectivement cette course à l'audience, on cherchait l'audience donc tous les moyens pour aller chercher les gens pour qu'ils viennent sur nos sites, quelle que soit la qualité de cette audience, la durée qu'ils allaient passer sur nos sites [...] c'était quand même une impression qu'on allait avoir et qu'on allait monétiser. On a dépassé complètement ce modèle [...] On va être plus dans des modèles où la partie diffusion/abonnement/vente de nos contenus est en train d'être beaucoup plus importante que les revenus qui sont liés à la pub donc ca ca change complètement le paradigme et ca veut dire qu'il faut créer de l'adhérence à nos contenus et sur nos sites, il faut créer de l'engagement sur nos sites, du temps d'attention. " (Les Echos-Le Parisien, 08/02/18)

Sans inviter explicitement les éditeurs à renoncer au financement publicitaire, Google semble appuyer leur constat d'échec de ce modèle de revenus. L' " assistance » de Google prend la forme d'un partenariat qui consiste à proposer aux éditeurs de devenir une brique technologique de leurs stratégies de recrutement d'abonnés en mobilisant ses technologies d'identification et de paiement (Subscribe with Google) ${ }^{6}$.

«Pour les médias, il y a plusieurs sources de revenus. Effectivement la publicité, mais aussi les abonnements et on voit lorsqu'on travaille avec les rédactions et les médias, à quel point il y a là un potentiel très riche. Google fait ce qu'il peut pour accompagner ce travail d'enrichissement de la base d'abonnés des médias. " (Google News Lab France, 14/03/18)

Les éditeurs de presse et la plateforme entretiennent un lien de dépendance mutuelle : l'accès 
aux publics est essentiel pour les éditeurs comme l'est la production de contenus d'actualités de qualité et diversifiée pour la plateforme.

\section{Google, point nodal de l'innovation dans le journalisme numérique}

Né en 2013, le Fonds de Soutien à l'Innovation Numérique de la Presse (FINP) concerne d'abord exclusivement la France (Israel, 2015). Il est doté de 60 millions d'euros pour une durée de trois ans. Ce fonds dédié à l'innovation éditoriale et commerciale est progressivement étendu et ses attributions sont élargies. En 2015, il devient une initiative européenne, la Digital News Initiative (DNI), avec 150 millions d'euros puis en mars 2018, la Google News Initiative (GNI) prend le relai avec une enveloppe de 300 millions de dollars sur trois ans ${ }^{7}$.

« Google ne réussit que si ses partenaires et en l'occurrence la presse, les médias, réussissent aussi. [...] la capacité que l'on a grâce à Google de les rechercher dépend de la richesse, de la variété, de la qualité de ces contenus et donc il est naturel pour Google, qui a pour mission d'organiser et de rendre accessible ces contenus, d'en favoriser la diversité et la qualité. " (directeur de Google News Lab France, 14/03/18)

Google souhaite jouer un rôle majeur dans les évolutions du journalisme numérique. Ainsi, le Google News Lab dédié à la conduite du changement dans les rédactions propose d'accompagner les éditeurs sur des sujets transversaux liés à l'innovation éditoriale et aux technologies émergentes en matière de fact-checking, de data journalisme, de machine learning ou d'intelligence artificielle.

Il existe trois catégories de projets d'innovation, auxquelles sont attribuées des budgets différents ${ }^{8}$. Les projets sont financés à hauteur de $70 \%$, les éditeurs doivent donc abonder une partie de la somme.

«Cette stimulation, ces nouvelles interactions avec le lecteur, ces changements de modèles économiques, on sait bien qu'ils viendront d'une diversité d'acteurs et c'est cette diversité qu'on veut encourager à travers ces appels à projets [...] on va regarder quel est l'impact sur l'écosystème, en quoi cela inspire les uns et les autres, en quoi cela peut avoir un bénéfice pour chacun parce que ca adresse une nouvelle façon de résoudre un problème ou d'essayer de le résoudre donc il ne s'agit pas de réinventer la roue à chaque fois, ça peut être une nouvelle façon d'aborder les choses. L'innovation nous on ne la définit pas, moi je ne sais pas exactement ce qu'est l'innovation, c'est les gens qui vont proposer un projet qui vont définir pourquoi c'est innovant » (Ludovic Blecher, responsable innovation GNI, 25/07/19).

Il s'agit là d'une forme d'externalisation de l'activité de Recherche \& Développement dans le secteur du journalisme. Google y joue pleinement le rôle de plateforme en s'appuyant sur sa modularité et l'enrôlement d'acteurs-tiers, en l'occurrence les éditeurs, pour capter la valeur que ceux-ci créent par leurs projets d'innovation. L'entreprise-en-réseau apprend ainsi à moindre coût, tout en distribuant les opportunités selon ses intérêts. Le financement par projet se fait en fonction de la pertinence que Google leur assigne. Il définit et ajuste librement le montant de cette aide privée et son périmètre, en restant maître de son investissement et des orientations de son fonds de soutien. Par définition, le financement par projet est limité car à court-terme, sélectif et concerne exclusivement une recherche appliquée. Google accepte de payer pour l'innovation, pas pour la production de contenus. Les entreprises de presse ne sont ni ses prestataires, ni ses fournisseurs et il ne souhaite pas en être le client. Ainsi, il tient à ce que les éditeurs apparaissent comme ses partenaires mais selon les termes et les conditions d'un contrat qu'il élabore seul.

«On n'est pas dans le soutien et l'accompagnement de l'existant, le but c'est de pouvoir identifier un projet précis qui permet de prendre des risques et de tester quelque chose que l'on n'aurait pas fait sans ce financement donc on cherche des projets autour de l'engagement avec le lecteur. L'engagement c'est comment augmenter la notoriété, le temps passé, l'audience à travers des logiques de personnalisation, à travers des logiques de nouvelles offres éditoriales et commerciales, comment on peut diversifier les revenus à travers des offres d'abonnement, à travers des newsletters, à travers de la registration, à travers diverses formes de diversification, donc engagement lecteur et nouveau modèle économique. Il faut être vraiment précis, on ne finance pas la création de contenus, ça ce n'est pas la proposition de GNI challenges, les entreprises de presse, les médias, les journalistes ont leur production de contenus, ça ca ne sera pas éligible. " (responsable innovation GNI, 25/07/19) 
Les éditeurs deviennent en partie dépendants de son assistance pour leurs développements technologiques stratégiques et la pérennité de leurs activités, reflétant une « capture infrastructurelle » du journalisme numérique (Nechushtai, 2017). Ceci étant, Google ne souhaite pas avoir à assumer le coût d'une dépendance, au sens où les éditeurs ne s'en remettraient qu'à la plateforme pour obtenir les moyens de leur subsistance. Par l'octroi d'une aide privée, l'entreprise a contribué à asseoir son emprise sur le journalisme, dans un contexte d'accroissement des difficultés économiques des éditeurs et de contraction de la dépense publique en soutien à la presse.

Google conditionne son financement à une procédure sélective nécessitant que les éditeurs fassent acte de candidature. Le formulaire de candidature au fonds de soutien pour l'innovation agit comme une " technologie intellectuelle " (Goody, 1979) ${ }^{9}$. Il participe à transformer l'état d'esprit des éditeurs de presse et exerce un pouvoir de normalisation sur les questions jugées pertinentes et les préoccupations devant être considérées comme stratégiques. Avec cet outil, Google modèle les représentations de ses " partenaires " selon son programme d'action à propos de ce qui compte, ce qui doit être jugé essentiel, ce qui est éventuellement négociable et ce qui ne l'est pas.

«On juge de la qualité de l’idée qui doit être précise et cohérente. On demande aux gens de nous proposer un budget, de nous dire pourquoi ils demandent tel ou tel montant et le projet doit avoir un angle précis et expliquer les opportunités. Sélectionné ou non, c'est une vraie opportunité de prendre un peu de recul par rapport aux opérations quotidiennes, d'essayer de casser un peu les silos, de prendre l'opportunité de réfléchir avec ses équipes et puis de penser à des choses que l'on n'aurait pas faites sans ce financement [...] La façon dont est construit le formulaire d'application est, je crois, assez intéressante et permet de réfléchir différemment autour de ces idées. " (responsable innovation éditeurs GNI, 25/07/19)

"On remplit un dossier extrêmement complexe, long, fastidieux à remplir avec des chiffres avant d'avoir le financement. On doit justifier pourquoi c'est un projet innovant, ça doit être un projet qui pour eux a du sens et qui est innovant parce qu'ils doivent le défendre en interne [...] D’une certaine manière, ils sont là pour nous aider et on veut aussi en profiter. On se dit [qu'] ils nous doivent quelque chose avec ce qui s'est passé avec la pub. On a peu d'infos sur ce qu'ils veulent vraiment, on ne sait pas ce qu'ils veulent vraiment faire [...] C'est des relations très compliquées, ils savent qu'on a besoin d'eux, qu'on est une industrie intéressante, c'est comment est-ce qu'on accepte de prendre ce qui nous nous intéresse et on s'y retrouve financièrement, en termes de financement de l'innovation, tout en faisant attention, mais c'est sûr qu'à chaque fois, c'est très délicat. " (responsable abonnement numérique Le Monde, 09/03/20)

L'asymétrie de pouvoir entre Google et les éditeurs se double donc d'une asymétrie d'informations qui la renforce. Grâce à son initiative, Google dispose d'une vision synoptique sur les difficultés de la presse. Cette dernière est, par ailleurs, alimentée par le financement d'études mondiales comparatives comme le Digital News Report du Reuters Institute for the Study of Journalism d'Oxford qui porte sur les évolutions de la consommation d'actualités numériques ${ }^{10}$. À cela, il convient d'ajouter l'analyse des données issues de l'usage de ses outils par les publics et les éditeurs. La connaissance ainsi accumulée lui permet d'enrichir son offre commerciale. L'ensemble de son initiative dans le journalisme garantit à Google d'identifier avant d'autres acteurs, et notamment grâce à la participation des éditeurs, les paris stratégiques sur lesquels se positionner très en amont. En maintenant partiellement privée cette connaissance, le géant californien joue un rôle pivot dans la circulation de l'information sur le marché, assurant ainsi une position centrale et endossant un rôle d'organisateur des échanges, de point nodal.

La presse ne peut se couper des publics de Google, ni des revenus afférents à cet apport de trafic. Elle cherche par conséquent à utiliser la force de son adversaire et de ses technologies pour améliorer ses affaires. Ceci ne l'empêche cependant pas de négocier la dépendance en cherchant un meilleur compromis avec l'appui de l'État.

\section{Partager le pouvoir : une déPendance négociée}

Google ne peut se passer de l'accès aux consommateurs européens. Le recours à une directive européenne pour créer le nouveau droit voisin des éditeurs de presse vise à obtenir une contrepartie financière en l'échange d'une autorisation d'exploitation des contenus. Le droit voisin s'inscrit dans un agenda politique français et européen de régulation des plateformes numériques dont le régime de responsabilité juridique est en cours d'élaboration. 
À travers l'affaire du droit voisin, nous observons la recherche d'un compromis dans la régulation du pouvoir des plateformes.

\section{L'européanisation du problème}

Définitivement adoptée par le Parlement européen le 26 mars 2019, la directive portant sur le droit d'auteur et le droit voisin participe de l'harmonisation réglementaire européenne dans le cadre du marché unique numérique (Viola et Bringer, 2017 ; Gaglio et Guillou, 2018). Entre 2017 et 2019, la Commission européenne s'est illustrée en infligeant trois amendes à Google pour un total de 8,25 milliards d'euros ${ }^{11}$. En particulier, c'est la commissaire européenne à la concurrence, Margrethe Vestager, qui a incarné cette politique de l'Europe envers les plateformes, devenue vice-présidente de la Commission européenne en 2019 chargée du numérique et de la concurrence.

Présenté à la Commission européenne en septembre 2016, le projet de « Directive sur le droit d'auteur dans le marché unique numérique » a pour objectif la révision de la directive sur le droit d'auteur et les droits voisins de 2001 afin de l'adapter au contexte numérique. Très contestée, la directive a donné lieu à des campagnes de communication et de mobilisation de la part de ses soutiens comme de ses opposants. Deux articles en particulier ont cristallisé les tensions : l'article 15 portant sur le droit voisin et l'article 17 sur le droit d'auteur.

L'article 15 instaure un nouveau droit voisin au profit des éditeurs et des agences de presse. Il leur permet d'autoriser contre rémunération la reproduction et la diffusion totale ou partielle de leurs contenus par les plateformes ${ }^{12}$. L'objectif premier de ce droit voisin est de remédier, par le droit de la propriété intellectuelle, à un déséquilibre économique dans la distribution des revenus publicitaires entre les plateformes et les éditeurs de presse. Alors que ces derniers ont à supporter des coûts de production élevés pour leurs contenus, ils dégagent des revenus numériques relativement modestes. Par comparaison, les plateformes ont à faire face à des coûts de production de leurs services bien inférieurs, répondant à de fortes économies d'échelles et des rendements croissants. De plus, elles engrangent l'essentiel de la valeur économique liée à la monétisation publicitaire des contenus. Le nouveau droit voisin propose donc l'ouverture de négociations collectives entre les plateformes et les éditeurs de presse afin de redistribuer une partie de la valeur des revenus publicitaires aux éditeurs de presse.
" Google a dit en gros : " on ne négociera jamais collectivement ". Ils préfèrent le modèle actuel [de négociations bilatérales], tout ce qui se passe en France maintenant, ca arrive en Europe et ensuite dans le monde [...] C'est vraiment, je trouve, une espèce de mondialisation, en tous cas d'européanisation de nos problèmes. Il y a encore 7,8 ans, on échangeait, [...] il y avait des différences, ce n'était pas tout à fait pareil suivant les pays, là globalement on a TOUS les mêmes problèmes, c'est-à-dire que tout le monde se fait bouffer la laine sur le dos par Google et Facebook. " (Directeur du pôle News Le Figaro et président du Geste, 23/01/20)

Le droit voisin a vocation à protéger les risques pris et les investissements consentis par les éditeurs de presse (Barraud, 2016), là où le droit d'auteur protège les contenus produits ${ }^{13}$. Le régime de protection juridique des éditeurs de presse par le droit voisin tient compte des spécificités du secteur de la presse et du rôle qu'elle joue dans le cadre d'une société démocratique $^{14}$. Pour l'heure, le droit s'est révélé inopérant à mettre en œuvre la politique escomptée de redistribution des gains et à inciter Google à des négociations collectives. L'entreprise américaine sait que les négociations avec les éditeurs français préfigurent celles avec les éditeurs européens. Elle a cependant commencé à attribuer le statut de "publications européennes de presse " à un certain nombre d'éditeurs.

\section{La presse épaulée par l'État}

La France est le premier pays européen à transposer l'article 15 de la directive européenne, le 24 juillet $2019^{15}$. Cette célérité s'explique par le volontarisme français en matière de régulation des plateformes numériques qui cherche à affirmer une vision francaise ${ }^{16}$. Dès le 5 septembre 2018, une proposition de loi est déposée par les sénateurs Assouline, Kanner et Daunis et les membres du groupe socialiste et républicain considérant être face à une situation d'urgence.

L'État français a souvent participé à encadrer la concurrence des éditeurs de presse, notamment en freinant l'extension de la publicité aux autres médias tels que la radio puis la télévision (Martin, 1988, 78). Il répond ainsi à l'appel à la régulation juridique des éditeurs de presse, après avoir activement et largement participé à documenter le déséquilibre du marché publicitaire numérique en faveur des plateformes et au détriment des éditeurs de presse par des études et des rapports parlementaires (Lescure, 2013 ; 
Franceschini, 2016 ; Adlc, 2018 ; BearingPoint, 2018 ; Mignola, 2019 ; Assouline, 2019 ; Adlc, 2019). La protection particulière de l'État dont bénéficie le secteur de la presse est fondée juridiquement et constitutionnellement, notamment en matière de pluralisme ${ }^{17}$. Les éditeurs de presse n'auraient « pas d'autres solutions que de développer leurs activités numériques pour assurer leur pérennité » face au déclin de la presse papier et à la diminution des revenus publicitaires associés ${ }^{18}$. Ainsi, le droit voisin serait appelé à jouer un "rôle stratégique " dans la «stabilité financière de la presse ». La publicité est considérée comme " une source de revenus vitale pour le secteur ", ce que soulignait déjà le rapport du député Mignola, repris dans un certain nombre d'articles de presse couvrant les événements, associant la survie économique des éditeurs de presse au pluralisme des médias et à la liberté de la presse. Le dispositif sur les droits voisins constituerait ainsi "une question de vie ou de mort » pour les médias (Adlc, 2020b).

La question n'est pas de savoir si les plateformes doivent être publiquement régulées mais plutôt comment elles doivent l'être (Gorwa, 2019). Les modalités de régulation des plateformes numériques sont au cœur de nombreuses propositions et rapports (FrisonRoche, 2019 ; Renaissance Numérique, 2020 ; Adlc, 2020a ; Digital New Deal, 2020) et en cours d'ajustements. L'État et les plateformes n'ont mutuellement pas intérêt à un affrontement, ils recherchent donc des formes de compromis débouchant sur un alignement de leurs intérêts (Tréguer, 2019). Ni l’État ni les éditeurs ne sont prêts à accepter la « capture infrastructurelle » (Nechushtai, 2017) de fait du journalisme sans compensation financière. Ils tentent donc d'élaborer un arrangement institutionnel avec la plateforme. Pour l'État, il s'agit de coopérer avec les plateformes, dans une dynamique partenariale, notamment à travers le droit de la conformité ou compliance (Frison-Roche, 2018 ; 2019) ${ }^{19}$. En échange de la levée de contraintes légales et d'une incertitude juridique pouvant peser sur leurs activités, les plateformes sont incitées à se conformer à une régulation sur-mesure, une " autorégulation sous contrôle » ou supervisée par l'État. Ce dernier espère ainsi mettre en œuvre une régulation " agile », plus rapide, réactive et effective, pouvant éventuellement inspirer un modèle européen de régulation des plateformes numériques.

\section{Du contournement de la règle de droit à l'injonction à négocier}

Un mois avant l'entrée en vigueur de la loi sur le droit voisin, Google a annoncé qu'il comptait modifier les règles d'affichage des contenus des éditeurs de presse :
«Lorsque la loi française entrera en vigueur, nous n'afficherons plus d'apercu du contenu en France pour les éditeurs de presse européens, sauf si l'éditeur a fait les démarches pour nous indiquer que c'est son souhait. Ce sera le cas pour les résultats des recherches effectuées à partir de tous les services de Google » (blog Google, 25/09/19)

De nouvelles "balises " ont été mises en place. Il s'agit de fragments de code que les éditeurs peuvent insérer dans le code source de leurs pages web afin d'autoriser Google à reprendre des extraits de leurs contenus éditoriaux sous forme de textes, d'images et de vidéos. Les trois balises "max-snippet ", " max-image-preview " et " max-video-preview " déterminent la longueur des extraits pouvant être indexés par les robots de Google. Le contournement de la règle de droit met en exergue la dimension asymétrique des rapports de dépendance entre les éditeurs et les plateformes. Elle démontre comment la plateforme gouverne par la régulation technique et l'architecture de son environnement ses interactions avec les éditeurs de presse. Sans jamais refuser explicitement de se soumettre au droit $^{20}$, la plateforme cherche à négocier une modalité de régulation plus souple et volontariste, non contraignante et individualisée avec certains éditeurs.

À la suite de l'action contentieuse lancée par les éditeurs de presse auprès de l'Adlc, des mesures conservatoires sont rendues. Une décision provisoire ordonne le 9 avril 2020 à Google de négocier collectivement avec les éditeurs de presse la rémunération de la reprise d'extraits de contenus dans un délai de trois mois et de revenir à la situation antérieure en matière d'affichage des contenus. L'Adlc souligne le « contournement " de la loi, les potentiels abus de dépendance économique et de position dominante $\mathrm{e}^{21}$. La politique de la concurrence ne remet pas en cause la position dominante de Google, ni n'altère le fonctionnement structurel de la relation entre Google et les éditeurs. Elle vise plutôt à transformer les modalités d'exercice du pouvoir de Google en l'assortissant de responsabilités vis-à-vis des éditeurs compte-tenu de son rôle infrastructurel. À travers la décision provisoire de l'Adlc, il est aussi rappelé à Google qu'il doit se résoudre à une négociation ouverte, transparente et collective plutôt qu'individualisée et discrétionnaire comme l'entreprise a toujours procédé jusqu'ici. Ainsi, Google se voit contraint de modifier sa manière de faire. Il ne peut décider seul et à discrétion des règles à appliquer, uniquement en fonction de ses intérêts. 


\section{ConcLusion}

Google participe avec ses services à mettre en forme la façon dont l'actualité est rendue visible aux publics. Il structure aussi la manière dont les éditeurs peuvent signaler leurs contenus et les véhiculer à travers une régulation par le code. De plus, il finance une initiative liée à l'innovation dans le journalisme numérique. Toutefois, Google ne peut gouverner seul. Il doit composer avec l'agenda politique français et européen de régulation des plateformes, dont le droit voisin n'est qu'un des aspects. À travers l'affaire du droit voisin, nous observons l'ajustement dynamique des modalités de régulation des plateformes. La recherche d'une effectivité rapide conduit l'État à partager son pouvoir de régulation des marchés, des échanges et des contenus numériques en accordant aux plateformes le rôle de partie prenante dans la régulation. Alors qu'il aurait pu choisir de limiter le pouvoir économique et politique des plateformes par une régulation externe, l'État en a fait des partenaires d'une régulation qu'il se contente de superviser. Il conviendra donc de continuer à être attentif à l'articulation de la régulation par les plateformes et de la régulation des plateformes par l'Europe et les États.

Les intérêts des éditeurs de presse et ceux des plateformes sont enchevêtrés et imbriqués d'un point de vue éditorial et commercial. La décision provisoire de l'Adlc précise que l'injonction à négocier ne devra pas affecter l'indexation, le classement, la présentation des contenus protégés repris sur les services de Google ou nuire aux relations économiques entre Google et les éditeurs de presse. Elle constitue un signal fort pour les autres pays européens et les éditeurs de presse du monde entier. Le droit peut et doit s'appliquer, en matière de propriété intellectuelle comme de concurrence, pour contraindre le pouvoir des plateformes à négocier un meilleur compromis social et économique à sa domination. Rompre avec la dépendance ne semble pas envisageable pour les éditeurs de presse car il y aurait trop à perdre : l'apport de trafic généré par le moteur de recherche, l'aide dans les projets de développement autour de l'innovation éditoriale et commerciale, l'appui enfin dans la monétisation publicitaire et le recrutement d'abonnés. Par conséquent, ils acceptent la dépendance en tentant d'en négocier les conditions et une contrepartie financière, épaulé en cela par le droit voisin et la politique de la concurrence. La dépendance des éditeurs aux plateformes n'est cependant ni totale ni définitive. Un compromis reste à établir et un arrangement institutionnel à trouver pour parvenir à une dépendance négociée acceptable pour tous.

Réception de l'article le 18 février 2020 Acceptation le 12 mai 2020 


\section{Notes}

${ }^{1 .}$ Hormis Google et Facebook, aucune entreprise ne capte une part des revenus du secteur de la publicité supérieure à $10 \%$ en France (Adlc, 2018, 47). Le duopole capte l'essentiel des revenus et de la croissance du marché de la publicité numérique, dont les relais sont le mobile, les réseaux sociaux et le programmatique sur lesquels les deux acteurs ont développé très précocement leurs positionnements stratégiques.

2. « La moyenne arithmétique du volume de trafic redirigé par Google pour les membres de l'APIG s'élève à plus de $42 \%$ ", dépassant ainsi le trafic direct (Adlc, 2020). L'Alliance de la Presse d'Information Générale (APIG) est une union de syndicats créée en septembre 2018 qui a pour mission de se constituer comme interlocuteur principal des pouvoirs publics et des acteurs privés. Elle représente l'intérêt collectif du secteur de la presse quotidienne nationale, régionale et départementale.

${ }^{3 .}$ Il s'agit d'un individu qui a l'intention d'acheter un véhicule automobile.

4. Le stack désigne l'ensemble des outils publicitaires Google, de l'hébergement à la mise en ligne des créations, en passant par l'achat d'espace, la diffusion et le suivi des retombées des campagnes publicitaires.

5. Un ad-exchange est une place de marché publicitaire automatisée qui réunit des offres (Supply-Side Platform ou SSP) et des demandes (Demand-Side Platform ou DSP), connectées à des plateformes de données (Data Management Platform ou DMP).

${ }^{6 .}$ Une commission de 5 à $15 \%$ est prélevée par l'intermédiaire (Rashidian et al., 2019)

${ }^{7}$ La somme de 300 millions de dollars peut paraître conséquente, mais le chiffre d'affaires mondial de l'entreprise Alphabet, maison-mère de Google, s'est élevé à près de 162 milliards de dollars en 2019 pour un bénéfice net de 34,3 milliards de dollars. En France, le chiffre d'affaires généré par Google dans la publicité numérique est supérieur à 2,5 milliards d'euros par an (cf. 23 $3^{\text {ème }}$ Observatoire de l'e-pub SRI-UDECAM, janvier 2020).

${ }^{8 .}$ Prototype jusqu'à $50000 €$, medium jusqu'à $300000 €$ et large jusqu'à 1 million d'euros. Voir aussi Mind Media, 29/03/19. " [Tableau] La liste des 75 projets français soutenus par Google DNI de 2016 à 2019 ».

${ }^{9}$ Le formulaire peut être librement téléchargé : https://newsinitiative.withgoogle.com/assets/static/docs/na-r2-challenge-questions.pdf

10. L'accord financier entre Google et l'Université d'Oxford pour une période de cinq ans entre août 2015 et août 2020 s'élève à 8,47 millions de pounds (soit plus de 9,6 millions d'euros). https://reutersinstitute.politics.ox.ac.uk/risj-review/google-anduniversity-oxford-agree-extension-support-digital-news-projectaugust-2020

11. En mars 2019, un montant d'1,49 milliards d'euros pour abus de position dominante (AdSense for search). En juillet 2018, 4,34 milliards d'euros pour " pratiques illégales " concernant les smartphones équipés du système d'exploitation Android et 2,42 milliards d'euros pour abus de position dominante avec le comparateur de prix Google Shopping en 2017. Google a systématiquement fait appel des amendes devant le Tribunal de la Cour de Justice de l'Union Européenne (CJUE).
${ }^{12 .}$ Le droit voisin dure jusqu'à deux ans après la publication de l'article. Une exception assez floue est prévue par le texte pour les contenus ne dépassant pas «quelques mots », Google l'interprète à son avantage en estimant que l'affichage du seul titre des articles n'ouvre pas droit à une rémunération.

13. Historiquement, le droit d'auteur a été conçu pour permettre à ceux qui sont à l'origine de la création de percevoir une partie des revenus qu'elle génère. Il est un outil de répartition de la richesse (Mazars, 2020). En ce sens, les finalités du droit d'auteur et du droit voisin sont proches.

14. Considérant 54 et 55 de la directive $n^{\circ} 2019 / 790$.

${ }^{15 .}$ Loi n ${ }^{\circ}$ 2019-775 du 24 juillet 2019 tendant à créer un droit voisin au profit des agences de presse et des éditeurs de presse.

URL: https://www.legifrance.gouv.fr/affichTexte.do? cidTexte=J ORFTEXT000038821358\& categorieLien=id.

La proposition de loi sur les droits voisins a été adoptée à l'unanimité au Sénat et à l'unanimité moins une voix à l'Assemblée nationale (voir aussi Mouron, 2019).

16. La Tribune, 12/03/20, "Big Tech": l'heure est à la responsabilité », par Olivier Sichel et Arno Pons de la Fondation Digital New Deal. Voir aussi le discours d'Emmanuel Macron prononcé le 12 novembre 2018 au Forum sur la gouvernance de l'internet à Paris

17. Conseil constitutionnel, décision n ${ }^{\circ} 86-210$ DC du 29 juillet 1986, Loi portant réforme du régime juridique de la presse, point 20

18. Entre 2007 et 2017, le secteur a perdu plus d'un tiers de son chiffre d'affaires, alors que la diffusion annuelle totale de la presse imprimée s'effondrait de $55 \%$. La baisse du chiffre d'affaires provient principalement des revenus issus de la publicité (-2,7 milliards d'euros, soit plus des deux tiers de la baisse totale de 4 milliards d'euros de chiffre d'affaires). Ces derniers représentent ainsi $31 \%$ du chiffre d'affaires total des éditeurs de presse en 2017 contre $44 \%$ en 2007. La baisse de la lecture de la presse écrite induit celle des revenus publicitaires (BearingPoint, 2018 ; Adlc, 2020b).

19. Les plateformes sont désignées par les notions de «plateforme numérique structurante " (Adlc, 2020a) ou d' "opérateur crucial » (Frison-Roche, 219). Cette distinction encourage une « régulation asymétrique », qui pourrait s'apparenter à une supervision de type prudentielle en continu et en temps réel, à l'instar de celle exercée sur les marchés financiers.

20. Il est à cet égard remarquable que le billet de blog de Google faisant l'annonce du changement des règles d'affichages s'intitule : « Nouvelles règles de droit d'auteur en France : notre mise en conformité avec la loi. » https://france.googleblog.com/2019/09/comment-nous-respectons-le-droit-dauteur. html

${ }^{21 .}$ La décision définitive sera prononcée en 2021. Le Monde, 09/04/20, « Droit d'auteur : Google sommé de négocier avec les médias pour rémunérer la reprise d'extraits de contenus. " 


\section{RÉFÉRENCES BIBLIOGRAPHIQUES}

Allary Jean et Vincent Balusseau, 2018, La publicité à l'heure de la data. Ad tech et programmatique expliqués par des experts, Paris : Dunod.

Arrese A., 2015, "From Gratis to Paywalls. A brief history of a retro-innovation in the press's business", Journalism Studies, Vol. 17, N8.

Assouline D., 2019, Rapport au Sénat $N^{\circ} 243$ sur la proposition de loi tendant à créer un droit voisin au profit des agences et des éditeurs de presse. 16 janvier, 78p.

Adlc, 2018, Avis n¹8-A-03, «Exploitation des données dans le secteur de la publicité sur internet », 6 mars, 125p.

Adlc, 2019, Avis n ${ }^{\circ}$ 19-A-04, relatif à une demande d'avis de la commission des Affaires culturelles et de l'Éducation de l'Assemblée national dans le secteur de l'audiovisuel, 21 février, 99p.

Adlc, 2020a, " Contribution de l'Autorité de la concurrence au débat sur la politique de la concurrence et les enjeux numériques ", 19 février, 14p.

Adlc, 2020b, Décision n ${ }^{\circ 20-M C-01, ~} 9$ avril, 72p.

Badouard R., C. Mabi et G. Sire, 2016, "Beyond "Points of Control": logics of digital governmentality", Internet Policy Review, Vol. 5, N³.

Barraud B., 2016, « Rapport de Laurence Franceschini sur la création d'un droit voisin pour les éditeurs de presse ", La revue européenne des médias et du numérique, $\mathrm{N}^{\circ} 40$, automne.

BearingPoint, 2018, Médias et Publicité en ligne. Transfert de valeur et nouvelles pratiques, Ministère de la Culture-CSA, 96p.

Bell E. \& T. Owen, 2017, The Platform Press: How Silicon Valley reengineered journalism, Tow Center for Digital Journalism.

Bucher T., 2012, "Want to be on the top? Algorithmic power and the threat of invisibility on Facebook", new media \& society, Vol. 14, $\mathrm{N}^{\circ} 7,1164-1180$.

Brown I. \& C.T. Marsden, 2013, "Regulating Code. Good Governance and Better Regulation in the Information Age”, Massachusetts: MIT Press.

Digital New Deal, 2020, « Réflexions dans la perspective du Digital Services Act européen ", Note d'étude, Liza Bellulo, mars 2020, 62p.

Digital News Report, 2019, Reuters Institute for the Study of Journalism, Oxford, 156p.

Dulong De Rosnay M., 2016, Les Golems du numérique. Droit d'auteur et Lex Electronica, Paris : Presses des Mines.

Franceschini L. et Bonnaud-Le-Roux S., 2016, Rapport de la mission de réflexion sur la création d'un droit voisin pour les éditeurs de presse, Conseil supérieur de la propriété littéraire et artistique, juillet 2016, 41p.

Frison-Roche M.A., 2018, « Droit de la concurrence et droit de la compliance ", Concurrences. Revue des droits de la concurrence, Éditorial, pp.1-4.

Frison-Roche M.A., 2019, « L'apport du droit de la compliance à la gouvernance d'internet », Rapport au Ministre en charge du Numérique, 139p.
Gaglio C. et S. Guillou, 2018, « L’Europe numérique. Entre singularités, faiblesses et promesses. ", Revue de l'OFCE, Vol. 4, N¹58, p.11-36

Goldsmith J. \& T. Wu, 2006, Who controls the internet. Illusions of a borderless world, Oxford: Oxford University Press. Goody J., 1979, La raison graphique, Paris : Minuit.

Gorwa R., 2019, "What is platform governance?”, Information, Communication \& Society, Vol. 22, $\mathrm{N}^{\circ} 6$ : AoIR Special Issue.

Guignard T., 2019, « Données personnelles et plateformes numériques : sophistication et concentration du marché publicitaire ", tic\&société, Vol. 13, n¹-2.

Grimmelmann J., 2005, "Regulation by Software", Yale Law Journal, N`114, pp. 1719.

Helmond A., 2015, "The Platformization of the Web: Making Web Data Platform Ready”, Social Media + Society, July-December, Vol. 1, $\mathrm{N}^{\circ} 2$, pp. 1-11.

Israel D., 2015, « Google et la presse : la raison du plus fort. Comment les journaux français ont fondu face au géant américain ", Revue du Crieur, 1, n 1, pp.66-81.

Lescure P., 2013, « Culture-acte2. Mission « Acte II de l'exception culturelle ». Contribution aux politiques culturelles à l'ère numérique ", Mai, 719p.

Lessig L., 1999, Code and other laws of Cyberespace, New York: Basic Books

Martin M., 1993, Trois siècles de publicité en France, Paris : Éditions Odile Jacob, 430p.

Martin M., 1988, « Le marché publicitaire Français et les grands médias 1918-1970 », Vingtième Siècle. Revue d'histoire, $\mathrm{N}^{\circ} 20$, Oct.-Dec., pp.75-90.

Mazars N., 2020, « Droit et rémunération des auteurs et des professionnels au niveau européen ", L'Observatoire, Vol. 1, n ${ }^{\circ} 55$, p.26-29.

Mignola P., 2019, Rapport №1912 Assemblée nationale, 30 avril, $96 \mathrm{p}$.

Mouron P., 2019, « Le nouveau droit voisin des éditeurs et agences de presse ", Revue européenne des Médias et du Numérique, n52, automne, pp.5-9.

Musiani F., 2013, "Governance by algorithms”, Internet Policy Review, Vol. 2, N³

Nechushtai E., 2017, "Could digital platforms capture the media through infrastructure?”, Journalism, Vol. 19, $\mathrm{N}^{\circ} 8$, pp. 1043-1058.

Plantin J.-C., C. Lagoze, P. N. Edwards and C. Sandvig, 2018, "Infrastructure studies meet platform studies in the age of Google and Facebook", New Media \& Society, Vol. 20, N¹, pp. 293-310.

Nielsen R. K. and S. A. Ganter, 2017, "Dealing with digital intermediaries: A case study of the relations between publishers and platforms”, New Media \& Society, Vol. 20, $\mathrm{N}^{\circ} 4$, pp.1600-1617.

Rashidian N., P. Brown, and E. Hansen with E. Bell, J. Albright \& A. Hartstone, 2018, Friend and Foe: The Platform Press at the Heart of Journalism; Tow Center for Digital Journalism. 
Rashidian N., G. Civeris, P. Brown with E. Bell \& A. Hartstone, 2019, Platforms and Publishers: The End of an Era, Tow Center for Digital Journalism, Columbia.

Renaissance Numérique, 2020, « Réguler les plateformes numériques : pourquoi ? comment? », Note, Mai, 25p.

Reidenberg J. R., 1998, "Lex Informatica: The Formulation of Information Policy Rules Through Technology", Texas Law Review, Vol. 76, N3, February, 43p.

Rieder B. and G. Sire, 2014, "Conflicts of interest and incentives to bias: A microeconomic critique of Google's tangled position on the Web", New Media \& Society, Vol. 16, N², pp.195-211.

Sire G., 2013, « La production journalistique et Google : chercher à ce que l'information soit trouvée ", Thèse de doctorat en Sciences de l'information et de la Communication, Université Panthéon Assas, 518p.

Smyrnaios N. \& F. Rebillard, 2019, "How infomediation platforms took over the news: a longitudinal perspective", The Political Economy of Communication, Vol. 7, $\mathrm{N}^{\circ} 1$, pp. 30-50.

Suzor N., 2019, Lawless: the secret rules that govern our digital lives (and why we need new digital constitutions that protect our rights), Cambridge: Cambridge University Press, 290p.

Tréguer F., 2019, L’utopie déchue. Une contre-histoire d'Internet. XVe-XXIe siècle, Paris : Fayard.
Viola R. et O. Bringer, 2017, «Vers un marché unique numérique : faire de la révolution numérique une opportunité pour l'Europe ", Revue d'économie financière, Vol. $1, \mathrm{~N}^{\circ} 125$, pp.239-254.

Ziewitz M., 2016, “Governing Algorithms: Myth, Mess and Methods”, Science, Technology \& Human Values, vol. 41, $\mathrm{N}^{\circ} 1$, pp. 4-16. 


\title{
Résumé | Abstract | Resumo
}

\author{
Négocier la dépendance ? \\ Google, la presse et le droit voisin
}

Negotiating Interdependence?

Google, the Press and Neighboring Rights

Negociando a dependência?

Google, a imprensa e os direitos conexos

Fr.

La relation entre les éditeurs de presse et Google peut être qualifiée de dépendance mutuelle, bien qu'elle s'inscrive dans un rapport de force asymétrique. Cette dépendance est à la fois économique et éditoriale. Google participe avec ses services à mettre en forme la façon dont l'actualité est rendue visible aux publics. À travers une régulation par le code, il structure aussi la manière dont les éditeurs balisent leurs contenus et les véhiculent. De plus, il finance une initiative liée à l'innovation dans le journalisme numérique dont plusieurs éditeurs de presse ont déjà bénéficié. Ces derniers contestent cependant le partage de la valeur lié à l'exploitation de leurs contenus numériques. Le nouveau droit voisin vise à remédier, par le droit de la propriété intellectuelle, au déséquilibre économique dans la distribution des revenus publicitaires entre les plateformes et les éditeurs de presse. En échange d'une autorisation d'exploitation des contenus, les plateformes doivent rémunérer les éditeurs de presse, marquant ainsi le passage d'une exploitation de fait à une exploitation de droit. À travers l'affaire du droit voisin, nous observons l'ajustement dynamique des modalités de régulation des plateformes, conduisant l'État à accorder à celles-ci le rôle de partie prenante dans la régulation. Pour cela, nous revenons sur deux faits qui ont suivi l'entrée en vigueur de la loi : la modification des règles d'affichage des contenus sur les services de Google et la décision provisoire de l'Autorité de la Concurrence. Si Google a cherché à échapper à la négociation collective et à la rémunération des éditeurs prévues par le droit voisin, la décision prononcée par l'Autorité l’oblige à négocier avec les éditeurs. Un compromis reste à établir et un arrangement institutionnel à trouver entre l'État, les éditeurs et les plateformes afin de négocier une dépendance acceptable.

Mots-clés : Google, presse, plateforme, régulation, numérique

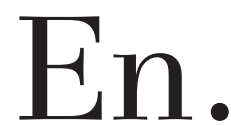

The relationship between press publishers and Google can be described as interdependent and asymmetrical. This interdependence is both economic and editorial. With its services, Google participates in shaping how news is made visible to the public, and through regulation by algorithms, it also structures the way publishers tag their content and convey it. Moreover, Google is funding an initiative targeting innovation in online journalism from which several press publishers have already benefited. However, these press publishers also contest how the value linked to the exploitation of their online content is shared. The new neighboring rights legistaltion aims to find a solution, through intellectual property laws, to the economic imbalance in the distribution of advertising revenues between platforms and press publishers; in exchange for an authorization to exploit content, platforms would pay press publishers, thus marking the transition from de facto exploitation to legal exploitation. With neighboring rights, a dynamic adjustment of the methods of regulating platforms is taking place, which has lead the French government to afford them a role of stakeholder in regulation. In this context, we analyze two events that followed the enactment of the neighboring rights legislation: the modification of the rules for the display of content on Google services and the provisional decision of the French Competition Authority (l'Autorité de la Concurrence). If Google has sought to evade 
collective bargaining and the remuneration of publishers provided for by neighboring rights legislation, the decision pronounced by the Authority compels the company to negotiate with publishers. A compromise remains to be established and an institutional arrangement to be found between the French government, publishers and platforms to negotiate an acceptable interdependence.

Keywords: Google, press publishers, platform, regulation, digital

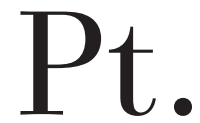

A relação entre os editores de jornais e a Google pode ser qualificada como de dependência mútua, ainda que assimétrica. Trata-se de uma dependência tanto econômica quanto editorial. A Google interfere com seus serviços no modo como as notícias se tornam visíveis para o público. Por meio de uma regulação pelo código, também estrutura a forma como os editores delimitam seu conteúdo e o transmitem. Além disso, a Google tem patrocinado uma iniciativa de inovação no jornalismo digital, da qual vários editores de jornais já se beneficiaram. Entretanto, esses editores têm contestado o compartilhamento do valor associado à exploração de seus conteúdos digitais. O novo direito conexo visa compensar, recorrendo ao direito de propriedade intelectual, o desequilíbrio econômico na distribuição das receitas publicitárias entre plataformas e jornais. Em troca de uma autorização de exploração de conteúdo, as plataformas devem remunerar os editores de imprensa, marcando assim a transição da exploração de fato para a exploração de jure. O caso dos direitos vizinhos revela como o ajuste dinâmico das modalidades regulatórias das plataformas leva o Estado a lhes outorgar o papel de parte interessada na regulação. Para tanto, focamos em dois fatos que acompanharam a entrada em vigor da lei: a modificação das regras de exibição de conteúdo nos serviços da Google e a decisão provisória da Autoridade da Concorrência. A Google buscou evitar a negociação coletiva e a remuneração dos editores prevista pela lei conexa, mas a decisão proferida pela Autoridade obrigou o grupo a negociar com os editores. Não se chegou ainda a nenhum acordo, nem arranjo institucional entre o Estado, os editores e as plataformas quanto a uma dependência aceitável.

Palavras-chave: Google, imprensa, plataforma, regulação, digital

\section{\&ิ)}

